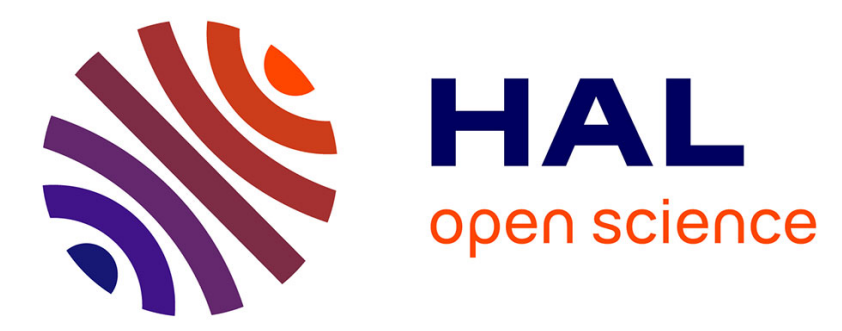

\title{
Global Asymptotic stabilization for some nonlinear models of flexible Aerospace vehicles
}

\author{
Laurent Burlion, Tarek Ahmed-Ali, Elodie Duraffourg, Françoise
}

Lamnabhi-Lagarrigue

\section{- To cite this version:}

Laurent Burlion, Tarek Ahmed-Ali, Elodie Duraffourg, Françoise Lamnabhi-Lagarrigue. Global Asymptotic stabilization for some nonlinear models of flexible Aerospace vehicles. 12th European Control Conference (ECC 2013), Dec 2013, Florence, Italy. pp.1-6, 10.1109/CDC.2013.6760539 . hal-00925415

\section{HAL Id: hal-00925415 \\ https://hal-centralesupelec.archives-ouvertes.fr/hal-00925415}

Submitted on 6 Jul 2021

HAL is a multi-disciplinary open access archive for the deposit and dissemination of scientific research documents, whether they are published or not. The documents may come from teaching and research institutions in France or abroad, or from public or private research centers.
L'archive ouverte pluridisciplinaire HAL, est destinée au dépôt et à la diffusion de documents scientifiques de niveau recherche, publiés ou non, émanant des établissements d'enseignement et de recherche français ou étrangers, des laboratoires publics ou privés.

\section{(c)(1)}

Distributed under a Creative Commons Attribution| 4.0 International License 


\title{
Global asymptotic stabilization for some nonlinear models of flexible aerospace vehicles
}

\author{
Laurent Burlion $^{1}$, Elodie Duraffourg ${ }^{1}$, Tarek Ahmed-Ali ${ }^{2}$ and Françoise Lamnabhi-Lagarrigue ${ }^{3}$
}

\begin{abstract}
New strategies are proposed to design GAS controllers for two significant classes of nonlinear flexible systems. Inspired by a non affine control technique, we have designed some novel nonlinear control laws which use the full state in order to enhance the damping of the flexible modes: this new design is all the more interesting than the classes of considered systems belong to non triangular nonlinear systems. Finally, the effectiveness of our approach is illustrated on simplified models of a flexible launcher and spacecraft.
\end{abstract}

\section{INTRODUCTION}

In the last years the stabilization problem of flexible nonlinear systems has received a great attention. This interest is motivated by the fact that flexible modes can generate a very bad transient even if the system is a minimumphase one. Several results concerning this topic exist in the literature : a typical design is to combine a nominal nonlinear controller with a vibration absorber controller.

This 'vibration absorber' is often based on the well known method of Input Shaping [1], [2], [3], [4] which has the major advantage to require merely relatively good approximation of modeling coefficients instead of direct measurements of the flexible modes : however, this useful method is by nature a linear method which can be used when the initial conditions are known and when the interaction between the flexible state and rigid ones is linear (although a few results exist in the nonlinear case e.g [5]). Another approach is to combine a nonlinear controller with a linear controller which uses the flexible modes and (only) locally damps them out [6], [7]. Open loop or robust feedforward methods can also be used to design a reference trajectory for the rigid state such that the oscillations of the flexible modes remain relatively low $[8],[5],[9]$ but it seems tricky to apply these methods on a large nonlinear operating domain when the systems parameters are uncertain and when there is a lot of flexible modes.

Recently, new nonlinear Lyapunov based approaches have been proposed for the stabilization problem of flexible aerospace systems when there is a large number of flexible modes and a strong nonlinear coupling between flexible and rigid modes $[10],[11],[12],[13]$ : technically speaking, although these systems are minimum phase, they belong to the

\footnotetext{
${ }^{1}$ L. Burlion and E. Duraffourg are with Onera - The French Aerospace Lab, F-31055 Toulouse, France. Laurent.Burlion (at) onera.fr Elodie.Duraffourg (at) onera.fr

${ }^{2}$ T. Ahmed-Ali is with Ecole Nationale Supérieure d'Ingénieurs de Caen (ENSICAEN), 6 boulevard du Marchal Juin 14050 Caen Cedex 4, France. tarek.ahmed-ali (at) greyc.ensicaen.fr

3 F. Lamnabhi-Lagarrigue is with L2S-CNRS, Supélec, 3 rue Joliot Curie, 91192 Gif-sur-Yvette, France. lamnabhi (at) lss.supelec.fr
}

class of underactuated non triangular nonlinear systems [15], which renders tedious the twofold objective of stabilizing the system and damping out the oscillations of the flexible modes. In this paper, we propose to enlarge these results by either adding more damping to the flexible dynamics $[10],[12],[13]$ either studying larger classes of systems [11]. The control schemes developed in this paper are based on the result of [16] which is a systematic design approach to add Lyapunov decrease to non affine nonlinear systems whose free dynamics are stable. Moreover, the major difference of our approach compared to classical methods is the fact that the controllers are first designed to stabilize the flexible modes (i.e the zero dynamics) and then extended to stabilize the full state.

The present paper is organized as follows : in section II, the class of nonlinear systems studied here are presented and motivated by some examples of flexible nonlinear systems. Then, we briefly recall the result of [16] used to determine the new control laws proposed in this paper : we show how one can both globally stabilize these classes of systems and add damping to the flexible dynamics. The two following sections illustrate our results on a flexible launch vehicle and a spacecraft model. We finally give our conclusions and some future research directions.

\section{A. Notations}

Let $m \in \mathscr{N}$. Throughout the paper, the following notations will be used:

- $\|$.$\| is the Euclidean norm in \mathscr{R}^{m}$

- Given a smooth function $V: \mathscr{R}^{2 m} \longrightarrow \mathscr{R}$ and a vector field $g: \mathscr{R}^{2 m} \longrightarrow \mathscr{R}^{2 m}, L_{g} V:=\frac{\partial V}{\partial x} g$ denotes the Liederivative of $V$ with respect to $g$

- a continuous function $\sigma: \mathscr{R}_{>0} \longrightarrow \mathscr{R}_{>0}$ belongs to class $\mathscr{K}$ it is monotonic increasing and $\sigma(0)=0$

- a continuous function $\sigma$ of class $\mathscr{K}$ belongs to class $\mathscr{K}_{\infty}$ if it is unbounded

- given a symmetric matrix $P$, its minimal (resp. maximal) eigenvalue is denoted by $\lambda_{\min }(P)$ (resp. $\lambda_{\max }(P)$ )

\section{PROBLEM FORMULATION}

In this paper, we consider the stabilization problem of the following classes of minimum-phase nonlinear systems :

A. Class I

$$
\left\{\begin{array}{l}
\dot{x}_{1}=x_{2} \\
\dot{x}_{2}=u \\
\dot{\eta}_{1}=\eta_{2} \\
\dot{\eta}_{2}=A_{\eta} \eta+B_{\eta} u+P_{\eta}^{1}\left(x_{1}\right)+P_{\eta}^{2}\left(x_{1}\right) x_{2}
\end{array}\right.
$$


This class of systems satisfies the following properties :

- $x_{1}, x_{2}, u \in \mathscr{R}$ and $\eta:=\left[\eta_{1} ; \eta_{2}\right] \in \mathscr{R}^{2 m}, m \in \mathscr{N}$

- $\left[\begin{array}{cc}0 & I_{m} \\ A_{\eta}\end{array}\right]$ is a real Hurwitz matrix and its eigenvalues are all complex conjugates

- $\eta_{1}$ is a vector whose coordinates are called the flexible modes

- the dynamics of $x=\left[x_{1} ; x_{2}\right]$ (resp. $\left[\eta_{1} ; \eta_{2}\right]$ ) is called the rigid (resp. flexible) dynamics

- all the $e_{i}^{T} P_{\eta}^{1}, i \in\{1, m\}$ are polynomials of degrees $\leq$ $d_{1}$, where $d_{1} \in \mathscr{N}$. Moreover, each $e_{i}^{T} P_{\eta}^{1}, i \in\{1, m\}$ vanishes at 0 .

- all the $e_{i}^{T} P_{\eta}^{2}, i \in\{1, m\}$ are polynomials of degrees $\leq d_{2}$ , where $d_{2} \in \mathscr{N}$

\section{B. Class II}

$$
\left\{\begin{array}{l}
\dot{x}_{1}=x_{2} \\
\dot{x}_{2}=u \\
\dot{\eta}_{1}=\eta_{2} \\
\dot{\eta}_{2}=A_{\eta} \eta+B_{\eta} u+f_{\eta}\left(x_{1}\right)+g_{\eta}\left(x_{1}, \eta_{1}\right) x_{2}^{2}
\end{array}\right.
$$

which verifies the following properties:

- $x_{1}, x_{2}, u \in \mathscr{R}$ and $\eta:=\left[\eta_{1} ; \eta_{2}\right] \in \mathscr{R}^{2 m}, m \in \mathscr{N}$

- $\left[\begin{array}{cc}0 & I_{m} \\ A_{\eta}\end{array}\right]$ is a real Hurwitz matrix and its eigenvalues are all complex conjugates

- $\eta_{1}$ is a vector whose coordinates are called the flexible modes

- the dynamics of $x=\left[x_{1} ; x_{2}\right]$ (resp. $\left[\eta_{1} ; \eta_{2}\right]$ ) is called the rigid (resp. flexible) dynamics

- $f_{\eta}\left(\right.$ resp. $\left.g_{\eta}\right)$ is a smooth and globally $k_{f}$-(resp. $\left.k_{g}\right)$ Lipschitz function and all the $e_{i}^{T} P_{\eta}, i \in\{1, m\}$ are polynomials of degrees $\leq l$, where $l \in \mathscr{N}$

It has to be noticed that there exist several flexible aerospace vehicles which are described by the above two classes of nonlinear systems. We can cite for instance :

- the rotational dynamics of a flexible hypersonic vehicle belongs to class $I$ (see for more details the modeling in [17] and some changes of coordinates leading to the class $I$ form in [11]).

- the longitudinal dynamics of a flexible launch vehicle belongs to class $I$ (see [18])

- the model of a spacecraft with a flexible attachment belongs to class II (see [14])

- the rotational dynamics of space vehicles with propellant sloshing mode belongs to class II (see [12], [13] when $a_{x}=a_{z}=0$ )

It is well-known that if the zero dynamics of the above systems are supposed asymptotically stable and by considering the state $x_{1}$ as output, then there exist several classical methods(e.g Backstepping [19] and Input/Output Linearization [20]), which solve the global asymptotic stabilization problem of these classes of systems. The most important drawback of these methods is that they do not integrate the zero dynamics in the design of the proposed controllers. This fact generates bad performances of the zero dynamics transients, which means that the flexible modes of several aerospace vehicles will exhibit some undesired oscillations. In the present work, we shall design some nonlinear controllers which simultaneously ensure a global asymptotic stability in closed loop and also improve the transient dynamics of the flexible modes. The major difference of the proposed schemes compared to the classical methods is in the fact that the controller is at first designed to stabilize the zero dynamics. This fact induces an improvement of the flexible modes behavior quality.

\section{MAIN RESULTS}

Before presenting our main results, we need to give the following important technical result which is largely derived from the work of [16] :

Lemma 1[Wei Lin, [16]] : Consider a single-input non affine nonlinear system of the following form :

$$
\dot{z}=f_{0}(z)+g_{0}(z) u+\sum_{i=2}^{l} g_{i}(z) u^{i}
$$

where $u \in \mathscr{R}, g_{i}: \mathscr{R}^{2 m} \longrightarrow \mathscr{R}^{2 m}, 2 \leq i \leq l$, are smooth functions. Suppose the unforced dynamics $f_{0}$ is such that there exists a function $V: \mathscr{R}^{2 m} \longrightarrow \mathscr{R}, \alpha_{0}$ of class $\mathscr{K}$, and $\alpha_{1}, \alpha_{2}$ of class $\mathscr{K}_{\infty}$ such that $\forall z$

$$
\begin{gathered}
\alpha_{1}(\|z\|) \leq V(z) \leq \alpha_{2}(\|z\|) \\
\dot{V}_{\mid u=0}=\frac{\partial V}{\partial z} f_{0}(z) \leq-\alpha_{0}(z)
\end{gathered}
$$

then the unforced system is GAS and the following control law

$$
u(z)=\varphi(z):=\frac{-\beta}{1+\|\partial V / \partial z\|^{2} \rho(z)^{2}} \frac{L_{g_{0}} V(z)}{1+\left(L_{g_{0}} V(z)\right)^{2}}
$$

where

$$
\rho(z) \geq \sum_{i=2}^{l} 1+\left\|g_{i}(z)\right\|^{2}
$$

and $\beta \in] 0,1[$ still renders system (3) GAS such that :

$$
\dot{V} \leq \dot{V}_{\mid u=0}-\frac{(1-\beta)}{1+\|\partial V / \partial z\|^{2} \rho(z)^{2}} \frac{\left(L_{g_{0}} V(z)\right)^{2}}{1+\left(L_{g_{0}} V(z)\right)^{2}} \leq \dot{V}_{\mid u=0}
$$

In this case, we say that $u$ adds damping to system (3) since it achieves more Lyapunov decrease.

Now, we are able to give the following propositions:

\section{A. Stabilization of Class I}

Proposition 1: let us consider the class of systems (1), then there exists a nonlinear feedback control law which simultaneously ensures the global asymptotic stability of the origin of the closed loop system and improves the transient of its corresponding zero dynamics.

\section{Proof:}


In order to prove the above proposition, let us consider the following change of coordinates:

$$
\left\{\begin{array}{l}
\bar{\eta}_{1}=\eta_{1}-B_{\eta} x_{1} \\
\bar{\eta}_{2}=\eta_{2}-B_{\eta} x_{2}
\end{array}\right.
$$

then, we obtain :

$$
\left\{\begin{array}{l}
\dot{x}_{1}=x_{2} \\
\dot{x}_{2}=u \\
\dot{\bar{\eta}}_{1}=\bar{\eta}_{2} \\
\dot{\bar{\eta}}_{2}=A_{\eta} \bar{\eta}+P_{\bar{\eta}}^{1}\left(x_{1}\right)+P_{\bar{\eta}}^{2}\left(x_{1}\right) x_{2}
\end{array}\right.
$$

where :

$$
\begin{aligned}
& P_{\bar{\eta}}^{1}\left(x_{1}\right):=P_{\eta}^{1}\left(x_{1}\right)+A_{\eta}\left[\begin{array}{c}
B_{\eta} \\
0
\end{array}\right] x_{1} \\
& P_{\bar{\eta}}^{2}\left(x_{1}\right):=P_{\eta}^{2}\left(x_{1}\right)+A_{\eta}\left[\begin{array}{c}
0 \\
B_{\eta}
\end{array}\right]
\end{aligned}
$$

From this, we can easily see that system (10) is a nontriangular nonlinear system non affine in $x_{1}$ but affine in $x_{2}$ according to the classification of underactuated nonlinear systems proposed in [15]. In order to remove $x_{2}$ in the flexible dynamics, let us use the following change of coordinates:

$$
\left\{\begin{aligned}
z_{1} & =\bar{\eta}_{1} \\
z_{2} & =\bar{\eta}_{2}-\left[\int_{0}^{1} P_{\bar{\eta}}^{2}\left(s x_{1}\right) d s\right] x_{1} \\
& :=\bar{\eta}_{2}-P_{\bar{\eta}}^{3}\left(x_{1}\right) x_{1}
\end{aligned}\right.
$$

Indeed, one can check that :

$$
\frac{d}{d t} P_{\bar{\eta}}^{3}\left(x_{1}\right) x_{1}=P_{\bar{\eta}}^{2}\left(x_{1}\right) x_{2}
$$

and that $P_{\bar{\eta}}^{3}\left(x_{1}\right) x_{1}$ is a polynomial of degree $\leq d_{2}$, then we obtain

$$
\left\{\begin{array}{l}
\dot{x}_{1}=x_{2} \\
\dot{x}_{2}=u \\
\dot{z}_{1}=z_{2}+P_{\bar{\eta}}^{3}\left(x_{1}\right) x_{1} \\
\dot{z}_{2}=A_{\eta} z+P_{\bar{\eta}}^{4}\left(x_{1}\right)
\end{array}\right.
$$

where

$$
P_{\bar{\eta}}^{4}\left(x_{1}\right)=A_{\eta}\left[\begin{array}{c}
0 \\
P_{\bar{\eta}}^{3}\left(x_{1}\right) x_{1}
\end{array}\right]+P_{\bar{\eta}}^{1}\left(x_{1}\right)
$$

is of degree $\leq \max \left\{d_{1}, d_{2}\right\}$.

Let us set $z=\left[z_{1} ; z_{2}\right]$. As we can see from (12), the zdynamics of the above system can now be viewed as a non affine triangular nonlinear system with respect to the input $x_{1}$. Since $\left[\begin{array}{cc}0 & I_{m} \\ A_{\eta}\end{array}\right]$ is Hurwitz, then there exists $P_{z}=P_{z}^{T}, Q_{z}>0$ such that :

$$
\left[\begin{array}{cc}
0 & I_{m} \\
A_{\eta}
\end{array}\right]^{T} P_{z}+P_{z}\left[\begin{array}{cc}
0 & I_{m} \\
A_{\eta}
\end{array}\right] \leq-Q_{z}
$$

We thus propose to use $V_{z}=z^{T} P_{z} z$ and we compute $\varphi$ according to (6) where :

$$
\begin{array}{rcc}
V & := & V_{z} \\
g_{0}(z) & = & g_{0}=\left[\begin{array}{c}
P_{\bar{\eta}}^{3}(0) \\
\frac{d}{d x_{1}} P_{\bar{\eta}}^{4}(0)
\end{array}\right] \\
g_{i}(z) & \stackrel{\forall i \in\{2, l\}}{=} & g_{i}=\left[\begin{array}{c}
(i-1) ! \frac{d^{i-1}}{d x_{1}^{i-1}} P_{\bar{\eta}}^{3}(0) \\
i ! \frac{d^{i}}{d x_{1}^{i}} P_{\bar{\eta}}^{4}(0)
\end{array}\right]
\end{array}
$$

We choose

$$
\delta_{1}=x_{1}-\varphi(z)
$$

Using (8) and the fact that :

$$
\forall i \in \mathscr{N}, \quad x_{1}^{i}=\left[\varphi+\delta_{1}\right]^{i}=\varphi^{i}+\sum_{k=0}^{i-1} C_{i}^{k} \varphi^{k} \delta_{1}^{i-k}
$$

then we have

$$
\begin{aligned}
\dot{V} \leq & -z^{T} Q_{z} z-\frac{(1-\beta)}{1+\|\partial V / \partial z\|^{2} \rho(z)^{2}} \frac{\left(L_{g_{0}} V(z)\right)^{2}}{1+\left(L_{g_{0}} V(z)\right)^{2}} \\
+ & {\left[\left(L_{g_{0}} V\right)+\sum_{\substack{2 \leq i \leq l \\
0 \leq k \leq i-1}} C_{i}^{k} \varphi^{k}\left(L_{g_{i}} V\right) \delta_{1}^{i-k-1}\right] \delta_{1} }
\end{aligned}
$$

Now, in order to design our controller, we shall use the classical Backstepping design [19] and consider the following Lyapunov function:

$$
W_{1}=V(z)+\frac{1}{2} \delta_{1}^{2}+\frac{1}{2} \delta_{2}^{2}
$$

where $\delta_{2}:=x_{2}-\bar{x}_{2}$ and

$$
\bar{x}_{2}=-k_{1} \delta_{1}+\dot{\varphi}-\left[\left(L_{g_{0}} V\right)+\sum_{\substack{2 \leq i \leq l \\ 0 \leq k \leq i-1}} C_{i}^{k} \varphi^{k}\left(L_{g_{i}} V\right) \delta_{1}^{i-k-1}\right]
$$

where $k_{1}>0$. If we choose the following control law :

$$
u=-\delta_{1}-k_{2} \delta_{2}+\frac{d}{d t}\left(\bar{x}_{2}\right)
$$

where $k_{2}>0$, then we obtain

$$
\begin{aligned}
\dot{W}_{1}= & -z^{T} Q_{z} z-\frac{(1-\beta)}{1+\|\partial V / \partial z\|^{2} \rho(z)^{2}} \frac{\left(L_{g_{0}} V(z)\right)^{2}}{1+\left(L_{g_{0}} V(z)\right)^{2}} \\
& -k_{1} \delta_{1}^{2}-k_{2} \delta_{2}^{2}
\end{aligned}
$$

which means the origin is GAS. This ends the proof.

\section{B. Stabilization of Class II}

Proposition 2: let us consider the class of systems (2) and define a bounded $C^{1}$ function $\chi$ and a positive constant $M_{\chi}>0$ such that $\forall x_{1}$,

$$
\left\{\begin{array}{l}
\left|\chi\left(x_{1}\right)\right| \leq M_{\chi} \min \left(\left|x_{1}\right|, 1\right) \\
x_{1} \chi\left(x_{1}\right) \leq-M_{\chi}\left\|x_{1}\right\|
\end{array}\right.
$$

then there exists a nonlinear feedback control law which simultaneously ensures the global asymptotic stability of the origin of the closed loop system and improves the transient of the corresponding zero dynamics.

\section{Proof:}

Let us give step by step the control law design:

1) Firstly, as for the above proposition, let us introduce a change of coordinates to eliminate $u$ from the flexible dynamics:

$$
\left\{\begin{array}{l}
\bar{\eta}_{1}=\eta_{1}-B_{\eta} x_{1} \\
\bar{\eta}_{2}=\eta_{2}-B_{\eta} x_{2}
\end{array}\right.
$$


in order to obtain a non linear system of the following form :

$$
\left\{\begin{array}{l}
\dot{x}_{1}=x_{2} \\
\dot{x}_{2}=u \\
\dot{\bar{\eta}}_{1}=\bar{\eta}_{2} \\
\dot{\eta}_{2}=A_{\eta} \bar{\eta}+\bar{f}_{\eta}\left(x_{1}\right)+\bar{g}_{2} x_{2}+\bar{g}_{\eta}\left(x_{1}, \bar{\eta}_{1}\right) x_{2}^{2}
\end{array}\right.
$$

where $\bar{g}_{2}:=A_{\eta}\left[\begin{array}{c}0 \\ B_{\eta}\end{array}\right], \bar{f}_{\eta}\left(x_{1}\right)=f_{\eta}\left(x_{1}\right)+A_{\eta}\left[\begin{array}{c}B_{\eta} \\ 0\end{array}\right]$ and $\bar{g}_{\eta}\left(x_{1}, \bar{\eta}_{1}\right)=g\left(x_{1}, \eta_{1}\right)$.

It is worth noting that such a system is a nontriangular nonlinear system non affine in $x_{1}$ and $x_{2}$ according to the classification of underactuated nonlinear systems proposed in [15].

2) Let us note $z=\left[x_{1} ; \bar{\eta}_{1} ; \bar{\eta}_{2}\right]$ and let us use the fact that $x_{2}=\chi\left(x_{1}\right)+\left(x_{2}-\chi\left(x_{1}\right)\right)$. Then, let us write the dynamics of $z$ :

$$
\dot{z}=f_{0}(z)+g_{0}(z)\left(x_{2}-\chi\left(x_{1}\right)\right)+g_{2}(z)\left(x_{2}-\chi\left(x_{1}\right)\right)^{2}
$$

where:

$$
\begin{aligned}
& f_{0}(z)=\left\{\begin{array}{l}
\chi\left(x_{1}\right) \\
\bar{\eta}_{2} \\
A_{\eta} \bar{\eta}+\bar{f}\left(x_{1}\right)+\bar{g}_{2} \chi+\bar{g}_{\eta}\left(x_{1}, \bar{\eta}_{1}\right) \chi^{2}
\end{array}\right. \\
& g_{0}(z)=\left\{\begin{array}{l}
1 \\
0 \\
\bar{g}_{2}+2 \bar{g}_{\eta}\left(x_{1}, \bar{\eta}_{1}\right) \chi\left(x_{1}\right)
\end{array}\right. \\
& g_{2}(z)=\left\{\begin{array}{l}
0 \\
0 \\
\bar{g}_{\eta}\left(x_{1}, \bar{\eta}_{1}\right)
\end{array}\right.
\end{aligned}
$$

3) then, given $P_{z}=P_{z}^{T}, Q_{z}>0$ satisfying (13), we propose:

$$
\begin{aligned}
V & =\log \left(1+\bar{\eta}^{T} P_{z} \bar{\eta}\right)+\frac{c_{1}}{2} x_{1}^{2} \\
& :=\log \left(1+V_{\bar{\eta}}\right)+\frac{c_{1}}{2} x_{1}^{2}
\end{aligned}
$$

where $c_{1}, c_{2}>0$ are given below.

From this we obtain :

$$
\begin{aligned}
& \dot{V}=\frac{-\bar{\eta}^{T} Q_{z} \bar{\eta}}{1+V_{\bar{\eta}}}+c_{1} x_{1} \chi\left(x_{1}\right) \\
& +2 \frac{\bar{\eta}^{T} P_{z}}{1+V_{\bar{\eta}}}\left(\bar{f}\left(x_{1}\right)+\bar{g}_{2} \chi\left(x_{1}\right)+\bar{g}_{\eta}\left(x_{1}, \bar{\eta}_{1}\right) \chi^{2}\left(x_{1}\right)\right) \\
& +\left(L_{g_{0}(z)} V\right)\left(x_{2}-\chi\left(x_{1}\right)\right)+\left(L_{g_{2}(z)} V\right)\left(x_{2}-\chi\left(x_{1}\right)\right)^{2}
\end{aligned}
$$

4) Using the fact that $2 \frac{\bar{\eta}^{T} P_{z}}{1+V_{\bar{\eta}}}=\frac{2 \bar{\eta}^{T} P_{z}^{1 / 2}}{1+\left(P_{z}^{1 / 2} \bar{\eta}\right)^{T}\left(P_{z}^{1 / 2} \bar{\eta}\right)} P_{z}^{1 / 2}$ and the assumptions on $\bar{f}, \bar{g}$, and $\chi$, then we have :

$$
\begin{aligned}
\left\|2 \frac{\bar{\eta}^{T} P_{z}}{1+V_{\bar{\eta}}} \bar{f}\left(x_{1}\right)\right\| & =\left\|\left(\frac{2 \bar{\eta}^{T} P_{z}^{1 / 2}}{1+V_{\bar{\eta}}}\right) P_{z}^{1 / 2} \bar{f}\left(x_{1}\right)\right\| \\
& \leq k_{f} \lambda_{\max }\left(P_{z}^{1 / 2}\right)\left\|x_{1}\right\| \\
\left\|2 \frac{\bar{\eta}^{T} P_{z}}{1+V_{\bar{\eta}}} \bar{g}_{2} \chi\left(x_{1}\right)\right\| & \leq M_{\chi}\left\|g_{2}\right\| \lambda_{\max }\left(P_{z}^{1 / 2}\right)\left\|x_{1}\right\|
\end{aligned}
$$

$$
\begin{aligned}
& \left\|2 \frac{\bar{\eta}^{T} P_{z}}{1+V_{\bar{\eta}}} \bar{g}_{\eta} \chi^{2}\left(x_{1}\right)\right\| \leq M_{\chi}^{2} \lambda_{\max }\left(P_{z}^{1 / 2}\right)\left\|x_{1}\right\| \\
& +2 k_{g} M_{\chi}\left\|x_{1}\right\|
\end{aligned}
$$

So, let us choose $c_{1}>0$ such that :

$c_{1}=\frac{\bar{c}_{1}+\left(k_{f}+M_{\chi}\left\|g_{2}\right\|+k_{g} M_{\chi}^{2}\right) \lambda_{\max }\left(P_{z}^{1 / 2}\right)+2 k_{g} M_{\chi}}{M_{\chi}}$

with $\bar{c}_{1}>0$, then we obtain,

$$
\begin{aligned}
\dot{V} \leq & \frac{-\bar{\eta}^{T} Q_{z} \bar{\eta}}{1+V_{\bar{\eta}}}-\bar{c}_{1}\left\|x_{1}\right\|+\left(L_{g_{0}(z)} V\right)\left(x_{2}-\chi\left(x_{1}\right)\right) \\
& +\left(L_{g_{2}(z)} V\right)\left(x_{2}-\chi\left(x_{1}\right)\right)^{2}
\end{aligned}
$$

5) then we use $x_{2}-\chi\left(x_{1}\right)=\left(x_{2}-\chi\left(x_{1}\right)-\varphi(z)\right)+\varphi(z)$ where $\varphi$ is given by (6). After a few computations, we obtain :

$$
\begin{aligned}
\dot{V} \leq & \frac{-\bar{\eta}^{T} Q_{z} \bar{\eta}}{1+V_{\bar{\eta}}}-\bar{c}_{1}\left\|x_{1}\right\| \\
& -\frac{(1-\beta)}{1+\|\partial V / \partial z\|^{2} \rho(z)^{2}} \frac{\left(L_{g_{0}} V(z)\right)^{2}}{1+\left(L_{g_{0}} V(z)\right)^{2}} \\
& +\left[\left(L_{g_{0}} V\right)+2 \varphi(z) L_{g_{2}} V+\left(L_{g_{2}} V\right) \delta_{2}\right] \delta_{2}
\end{aligned}
$$

where

$$
\delta_{2}:=x_{2}-\chi\left(x_{1}\right)-\varphi(z)
$$

6) finally, if we use the following Lyapunov candidate function :

$$
W_{2}=V+\frac{1}{2} \delta_{2}^{2}
$$

and by choosing the following control law :

$$
\begin{aligned}
u= & -k_{2}\left(x_{2}-\chi\left(x_{1}\right)\right)-\left(L_{g_{0}} V\right)-2 \varphi(z) L_{g_{2}} V \\
& -\left(L_{g_{2}} V\right) \delta_{2}+\frac{d}{d t}\left(\chi\left(x_{1}\right)+\varphi(z)\right)
\end{aligned}
$$

where $k_{2}>0$, we derive that :

$$
\begin{aligned}
\dot{V} \leq & \frac{-\bar{\eta}^{T} Q_{z} \bar{\eta}}{1+V_{\bar{\eta}}}-\bar{c}_{1}\left\|x_{1}\right\|-k_{2} \delta_{2}^{2} \\
& -\frac{(1-\beta)}{1+\|\partial V / \partial z\|^{2} \rho(z)^{2}} \frac{\left(L_{g_{0}} V(z)\right)^{2}}{1+\left(L_{g_{0}} V(z)\right)^{2}}
\end{aligned}
$$

from this we conclude that the origin of system (2) is GAS.

\section{ApPlicAtion to A NONLINEAR MODEL OF A FLEXIBLE LAUNCH VEHICLE}

\section{A. Mathematical Model}

From the full Mathematical model of a flexible launch vehicle obtained by using the Lagrange equations, we extract the rotational 'fast' dynamics (for more details, see ([18])):

$$
\left\{\begin{aligned}
\dot{\psi} & =q \\
\dot{q} & =-\frac{l_{\text {aero }}}{I_{L}} L(\psi)+\frac{T}{I_{L}}\left(L_{T} r-h\right) \eta_{1}+\frac{T L_{T}}{I_{L}} \beta \\
\ddot{\eta}_{1} & =-\omega^{2} \eta_{1}-2 \xi \omega \dot{\eta}_{1}+h r T \eta_{1}+h T \beta
\end{aligned}\right.
$$


where $\psi, \eta_{1}$ belong to $\mathscr{R}$ (We only consider the first flexible mode which is known to be the only one to be relevant to consider in the control design) and $\beta \in \mathscr{R}$ is the control. where the lift is a non-linear function of the attitude, given by:

$$
L(\psi)=\bar{q} S\left(C_{L}^{1} \psi+C_{L}^{2} \psi^{2}\right)=\bar{L}(\psi) \psi
$$

\section{B. Controller design}

First we use the notation :

$$
u:=-\frac{l_{\text {aero }}}{I_{L}} L(\psi)+\frac{T}{I_{L}}\left(L_{T} r-h\right) \eta_{1}+\frac{T L_{T}}{I_{L}} \beta
$$

By noting $x_{1}=\psi, x_{2}=q, \dot{\eta}_{1}=\eta_{2}$, we obtain a model which belongs to the Class $I$ :

$$
\left\{\begin{array}{l}
\dot{x}_{1}=x_{2} \\
\dot{x}_{2}=u \\
\dot{\eta}_{1}=\eta_{2} \\
\dot{\eta}_{2}=-\bar{\omega}^{2} \eta_{1}-2 \xi \omega \eta_{2}+\frac{h l_{\text {aero }}}{L_{T}} L\left(x_{1}\right)+\frac{h I_{L}}{L_{T}} u
\end{array}\right.
$$

with $\bar{\omega}^{2}:=\omega^{2}+\frac{h T}{L_{T}}\left(L_{T} r-h\right)-h r T$.

Applying the design method described in the proof of proposition 1 , then we obtain : the following dynamics :

$$
\left\{\begin{aligned}
\dot{x}_{1}= & x_{2} \\
\dot{x}_{2}= & u \\
\dot{z}_{1}= & z_{2}-2 \xi \omega \frac{h I_{L}}{L_{T}} x_{1} \\
\dot{z}_{2}= & -\bar{\omega}^{2} z_{1}-2 \xi \omega z_{2}+\frac{h}{L_{T}}\left(4 \xi^{2} \omega^{2} I_{L}-\bar{\omega}^{2} I_{L}\right. \\
& \left.+l_{\text {aero }} \bar{q} S C_{L}^{1}\right) x_{1}+\frac{h l_{\text {aero }}}{L_{T}} \bar{q} S C_{L}^{2} x_{1}^{2}
\end{aligned}\right.
$$

We then numerically solve the Lyapunov equation by considering the equality (instead of the inequality) associated to (13). We obtain :

$$
V=z^{T} P_{z} z
$$

we then compute $\varphi$ according to (6); in this case, we use :

$$
\begin{aligned}
g_{0} & =\frac{h}{L_{T}}\left[\begin{array}{c}
-2 \xi \omega I_{L} \\
4 \xi^{2} \omega^{2} I_{L}-\bar{\omega}^{2} I_{L}+l_{\text {aero }} \bar{q} S C_{L}^{1}
\end{array}\right] \\
g_{2} & =\frac{h}{L_{T}}\left[\begin{array}{c}
0 \\
l_{\text {aero }} \bar{q} S C_{L}^{2}
\end{array}\right]
\end{aligned}
$$

Then, given two gains $k_{1}, k_{2}>0$ the proposed controller is :

$$
u=-\left(x_{1}-\varphi(z)\right)-k_{2}\left(x_{2}-\bar{x}_{2}\right)+\frac{d}{d t} \bar{x}_{2}
$$

where:

$$
\begin{aligned}
\bar{x}_{2}= & -k_{1}\left(x_{1}-\varphi(z)\right)+\dot{\varphi}-L_{g_{0}} V \\
& -\left(L_{g_{2}} V\right)\left(x_{1}-\varphi(z)\right)-2 \varphi(z)\left(L_{g_{2}} V\right) \\
= & -k_{1}\left(x_{1}-\varphi(z)\right)+\dot{\varphi}-L_{g_{0}} V-\left(L_{g_{2}} V\right)\left(x_{1}+\varphi(z)\right)
\end{aligned}
$$

This law is compared to the following 'nominal' Backstepping law :

$$
\begin{aligned}
u_{0} & =-x_{1}-k_{2}\left(x_{2}-\bar{x}_{20}\right)+\frac{d}{d t} \bar{x}_{20} \\
& :=-x_{1}-k_{2}\left(x_{2}+k_{1} x_{1}\right)-\frac{d}{d t}\left(k_{1} x_{1}\right) \\
& =-\left(1+k_{1} k_{2}\right) x_{1}-\left(k_{1}+k_{2}\right) x_{2}
\end{aligned}
$$

\section{Numerical results}

The damping of the flexible mode is chosen very low (0.01) and the simulations are performed under the initial conditions $\psi(0)=20 \mathrm{deg}, q(0)=20 \mathrm{deg} / \mathrm{s}$ far from the origin.

Figure (1) shows in blue (resp. in orange) the evolution of both $\psi$ and $\eta_{1}$ when the controller $u$ (resp. $u_{0}$ ) is applied to the system. We observe that controller $u$ enhances the transient of the flexible mode.

\section{APPliCATION to A NONLINEAR MODEL OF A FLEXIBLE SPACECRAFT}

\section{A. Mathematical Model}

We consider the following model of a flexible attachment to a spacecraft which has been obtained after a partial feedback linearization [14]

$$
\left\{\begin{array}{l}
\dot{x}_{1}=x_{2} \\
\dot{x}_{2}=u \\
\dot{\eta}_{1}=\eta_{2} \\
\dot{\eta}_{2}=-\omega^{2} \eta_{1}-2 \xi \omega \eta_{2}+\eta_{1} x_{2}^{2}-\alpha u
\end{array}\right.
$$

\section{B. Controller design}

Applying the design method described in the proof of proposition 2, we first use the following change of coordinates :

$$
z=\left\{\begin{array}{l}
x_{1} \\
\bar{\eta}_{1} \\
\bar{\eta}_{2}
\end{array}:=\left\{\begin{array}{l}
x_{1} \\
\eta_{1}+\alpha x_{1} \\
\eta_{2}+\alpha x_{2}
\end{array}\right.\right.
$$

We then numerically solve the Lyapunov equation by considering the equality (instead of the inequality) associated to (13). We obtain :

$$
V_{\bar{\eta}}=\bar{\eta}^{T} P_{z} \bar{\eta}
$$

Then we choose (for instance)

$$
\chi\left(x_{1}\right)=-M_{\chi} \tanh \left(x_{1}\right)
$$

where $M_{\chi}>0$ and we rewrite the dynamics as follows :

$$
\begin{aligned}
\dot{z}= & f_{0}(z)+g_{0}(z)\left(x_{2}-\chi\left(x_{1}\right)\right)+g_{2}(z)\left(x_{2}-\chi\left(x_{1}\right)\right)^{2} \\
= & f_{0}(z)+g_{0}(z) \varphi(z)+g_{2}(z) \varphi^{2}+g_{0}(z) \delta_{2} \\
& v+2 g_{2}(z) \varphi(z) \delta_{2}+g_{2}(z) \delta_{2}^{2}
\end{aligned}
$$

we then compute $\varphi$ according to (6) ; in this case, we use :

$$
\begin{aligned}
& V= \log \left(1+V_{\bar{\eta}}\right)+\frac{1}{2} c_{1} x_{1}^{2} \\
& f_{0}=\left\{\begin{array}{l}
\chi\left(x_{1}\right) \\
\bar{\eta}_{2} \\
-\omega^{2} \bar{\eta}_{1}-2 \xi \omega \bar{\eta}_{2}+\omega^{2} \alpha x_{1} \\
+2 \xi \omega \alpha \chi\left(x_{1}\right)+\left(\bar{\eta}_{1}-\alpha x_{1}\right) \chi^{2}\left(x_{1}\right)
\end{array}\right. \\
& g_{0}=\left\{\begin{array}{l}
1 \\
0 \\
2 \xi \omega \alpha+2\left(\bar{\eta}_{1}-\alpha x_{1}\right) \chi\left(x_{1}\right) \\
0 \\
g_{2}=
\end{array}\right.
\end{aligned}
$$


Our control law is finally given by (41) and compared to the following 'nominal' Backstepping control law :

$$
\begin{aligned}
u_{0} & =-k_{2}\left(x_{2}-\chi\left(x_{1}\right)\right)+\dot{\chi}\left(x_{1}\right) \\
& =-k_{2}\left(x_{2}+M_{\chi} \tanh \left(x_{1}\right)\right)-M_{\chi}\left(1-\tanh ^{2}\left(x_{1}\right)\right) x_{2}
\end{aligned}
$$

\section{Numerical results}

In order to give more importance to the nonlinear term in $x_{2}^{2}$, we used a high coupling coefficient $\alpha=-1$ (by comparison to the very small value of a real model [14]). Moreover, the damping of the flexible mode is chosen very low (0.01) and the simulation model is run under the following initial conditions $x_{1}(0)=45 \mathrm{deg}, x_{2}(0)=0 \mathrm{deg} / \mathrm{s}$ far from the origin. Figure (2) clearly shows the damping enhancement of the flexible mode when we apply our novel controller (in blue).

\section{CONCLUSIONS}

New strategies have been proposed to design GAS controllers for two significant classes of nonlinear minimum phase systems. Inspired by a non affine control method [16], we have designed some novel nonlinear control laws which take into account the zero dynamics of the considered systems. In future work, we will extend our results to systems with uncertain parameters and unmeasured flexible states.

\section{FIGURES}
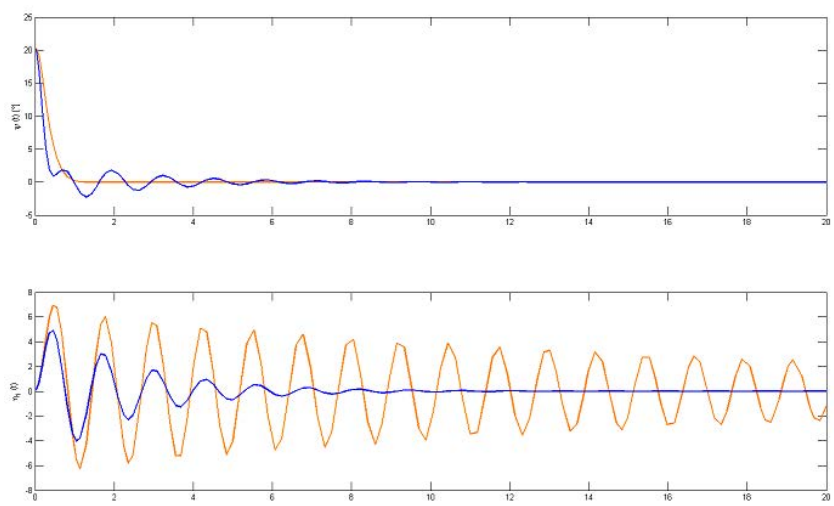

Fig. 1. Application 1 : evolution of $\psi(t)$ and $\eta_{1}(t)$ (below)

\section{REFERENCES}

[1] K.L. Sorensen, W.E. Singhose and S. Dickerson, A controller enabling precise positioning and sway reduction in bridge and gantry cranes, Control Engineering Practice, vol.15, no. 7, pp. 825-837, 2007.

[2] W. Aribowo, T. Yamashita, K. Terashima and H. Kitagawa, Input shaping control to suppress sloshing on liquid container transfer using multi-joint robot arm, IEEE International Conference on Intelligent Robots and Systems, pp. 3489-3494, 2010.

[3] B. Pridgen, K. Bai and W.E. Singhose, Slosh suppression by robust input shaping, IEEE International Conference on Decision and Control, pp. 2316-2321, 2010.

[4] Q. Hu, Z. Wang and H-J Gao, Sliding mode and shaped input vibration control of flexible systems, IEEE Transactions on Aerospace and Electronic Systems, pp. 503-519, 2008.
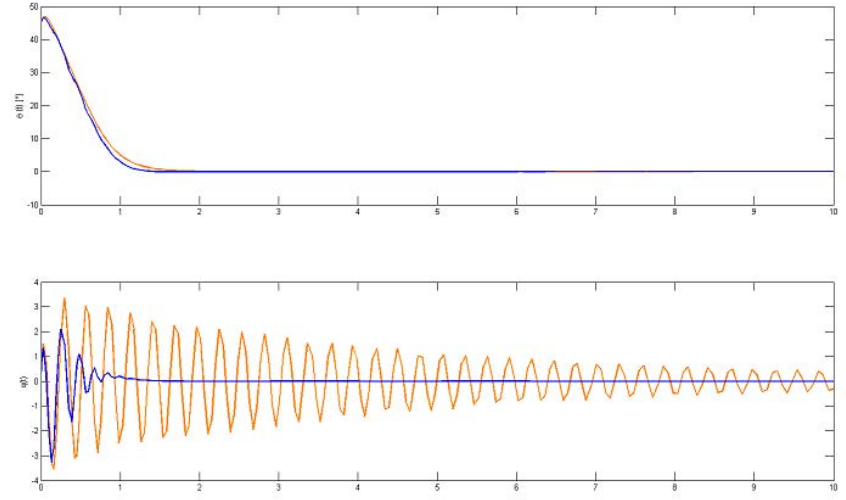

Fig. 2. Application 2 : evolution of $x_{1}(t)$ and $q(t)$ (below)

[5] D. Gorinevsky and G. Vukovichz, Nonlinear Input Shaping Control of Flexible Spacecraft Reorientation Maneuver, AIAA Journal of Guidance, Control, and Dynamics, vol. 21, no. 2, pp. 264-270, 1998.

[6] M. Malekzadeh, A. Naghash and H.A Talebi, Heidar, A Robust Nonlinear Control Approach for Tip Position Tracking of Flexible Spacecraft, IEEE Transactions on Aerospace and Electronic Systems, pp. 2423-2434, 2011.

[7] G. Bartolini, A. Pisano and E. Usai, Second-order sliding-mode control of container cranest, Automatica, pp. 17831790, 2002.

[8] K. Graichen and M. Zeitz, Feedforward control design for finite time transition problems of non-linear MIMO systems under input constraints, in International Journal of Control, vol. 81, no. 3, pp. 417427, 2008.

[9] I.A. Shkolnikov and Y.B. Shtessel, Tracking in a class of nonminimum-phase systems with nonlinear internal dynamics via sliding mode control using method of system center, Automatica, pp. 837-842, 2002.

[10] L. Fiorentini, A. Serrani, M. Bolender and D. Doman, Nonlinear Robust Adaptive Control of Flexible Air-Breathing Hypersonic Vehicles, Journal of Guidance, Control and Dynamics, vol. 32, no. 2, pp. 402417, 2009.

[11] E. Duraffourg, L. Burlion, T. Ahmed-Ali and F. Lamnabhi-Lagarrigue, Nonlinear control of the longitudinal rotational dynamics of a flexible aircraft, in Proc. European Control Conference, Zürich, pp. 335-340, 2013.

[12] M. Reyhanoglu and J. Hervas, Point-to-Point Liquid Container Transfer via a PPR Robot with Sloshing Suppression, in Proc. American Control Conference, Montréal, pp. 5490-5494, 2012.

[13] M. Reyhanoglu and J. Hervas, Nonlinear dynamics and control of space vehicles with multiple fuel slosh modes, Control Engineering Practice, vol. 20, pp. 912-918, 2012.

[14] M. Reyhanoglu, Slewing maneuver of a flexible spacecraft using finite time control, in Proc. of the 34th IEEE International Conference IECON, pp. 2667-2671, 2008.

[15] R. Olfati-Saber, Nonlinear Control of Underactuated Mechanical Systems with Application to Robotics and Aerospace Vehicles, PHD Thesis, MIT, Cambridge, MA, February 2001.

[16] W. Lin, Global asymptotic stabilization of general nonlinear systems with stable free dynamics via passivity and bounded feedback, Automatica, Volume 32, Issue 6, 1996, pp. 915-924, 1996.

[17] M. Bolender and D. Doman, Nonlinear Longitudinal Dynamical Model of an Air-Breathing Hypersonic Vehicle, Journal of Spacecraft and Rockets, vol.44, no. 2, pp. 374, 2007.

[18] E. Duraffourg, L. Burlion and T. Ahmed-Ali, Longitudinal Modeling and Preliminary Control of a Non-linear Flexible Launch Vehicle, in Proc. IFAC ALCOSP Conference, Caen, pp. 209-214, 2013.

[19] R. Sepulchre, M. Jankovic and P.V. Kokotovic, Constructive nonlinear control, Springer, 1997.

[20] A. Isidori, Nonlinear Control Systems , Springer, third edition, 1995. 